\title{
Disminución del riesgo de eventos cardiacos utilizando beta- bloqueantes en pacientes de alto riesgo sometidos a cirugía vascular
}

The effect of bisoprolol on perioperative mortality and myocardial infarction in high-risk patients undergoing vascular surgery. Poldermans D, Boersma E, Bax JJ, et al. for the Dutch Echocardiographic Cardiac Risk Evaluation Applying Stress Echocardiography Study Group. N Engl J Med 1999;341:1789-94.

\section{Objetivo}

Evaluar los efectos del bloqueo beta-adrenergico perioperatorio sobre la incidencia de muerte de causa cardiaca e infarto no fatal dentro de los 30 días posteriores a una cirugía vascular mayor (reconstrucción de aorta abdominal o de arterias infrainguinales) en pacientes con alto riesgo para esos eventos.

\section{Diseño}

Estudio clínico randomizado, no ciego con seguimiento a 30 días.

\section{Lugar}

Ensayo multicéntrico llevado a cabo en 8 centros europeos. Uno de Bélgica, 2 de Italia y 5 de Holanda.

\section{Pacientes}

Desde 1996 a 1999 se seleccionaron 112 pacientes con un resultado positivo del eco-stress con dobutamina sometidos a cirugía vascular mayor con 1 o más factores de riesgo cardiaco (edad > 70 años, angina, infarto previo, historia de insuficiencia cardiaca (IC) o IC compensada, tratamiento actual por arritmias cardiacas o diabetes y/o limitada capacidad de realizar actividades diarias normales) de una población total de 1351 pacientes.

\section{Intervención}

Se randomizó a los pacientes a recibir cuidados perioperatorios standard mas bisoprolol 5-10 mg/día (59 pac.) o únicamente cuidados standard (53 pac.). El tratamiento con bisoprolol fue iniciado un promedio de 37 días (rango 7 a 89) previo a la cirugía.

\section{Medición de resultados principales}

Se definió el punto final combinado del estudio a la muerte de causa cardiaca y el infarto no fatal durante el periodo perioperatorio.

\section{Resultados principales}

Dos pacientes en el grupo de tratamiento con bisoprolol murieron de causa cardíaca (3.4\%) comparado con 9 pacientes en el grupo de cuidados standard $(17 \%, p=0.02)$. Nueve pacientes tuvieron un infarto no fatal en el grupo de cuidados standard (17\%) y ninguno del grupo de cuidado estándar mas bisoprolol $(p<0.001)$. La frecuencia global de eventos combinados fue de 34\% (IC 95\% 21-48\%) en el grupo de cuidados standard comparado con 3.4\% (IC 95\% 0-8.0\% p <0.001) en el grupo de cuidados estándar más bisoprolol. El riesgo relativo de muerte de causa cardiaca o infarto no fatal durante el periodo perioperatorio entre aquellos pacientes que recibieron bisoprolol versus tratamiento standard fue RR 0.09 IC 95\% 0.02-0.37, p <0.001. La diferencia significativa en la incidencia de eventos entre los grupos en un análisis intermedio hizo que el comité de seguridad recomendara interrumpir el estudio.

\section{Conclusión}

El uso de bisoprolol reduce la incidencia perioperatoria de muerte de causa cardiaca e infarto no fatal en pacientes de alto riesgo sometidos a cirugía vascular mayor.

\section{COMENTARIO}

La evaluación cardiovascular de los pacientes candidatos a cirugía no cardiaca ha sido intensamente investigada en los últimos años siendo esta evidencia la base para la elaboración de las guías publicadas por el American College of Cardiology y la American Heart Association. ${ }^{1}$ Sin embargo el énfasis ha sido mayormente orientado a como identificar pacientes en mediano y alto riesgo y poco se conoce acerca de las estrategias que podrían mejorar su evolución. Solo un estudio randomizado evaluó el efecto cardioprotector de los beta-bloqueantes en este escenario y demostró una reducción en la mortalidad a 2 años del $55 \%$ en aquellos pacientes tratados con atenolol versus placebo. ${ }^{2} \mathrm{El}$ mecanismo por el cual los beta-bloqueantes reducen los eventos cardiacos no es del todo claro pero estaría relacionado al efecto sobre la frecuencia cardiaca y la contractilidad miocárdica, de manera tal que su uso evita un aumento de la demanda de oxigeno y con esto impide el desarrollo de isquemia miocárdica. Es importante enfatizar que los eventos coronarios no fatales en pacientes hospitalizados que son sometidos a cirugía no cardiaca esta asociado con un elevado riesgo de complicaciones precoces y tardías. Estudios de este grupo de pacientes han mostrado que aquellos que padecían un infarto no fatal en el periodo postoperatorio precoz de cirugía vascular tienen 4 veces más riesgo de tener un infarto fatal o no fatal en los próximos 4 años, subrayando la importancia de la isquemia postoperatoria como predictor de evolución a largo plazo.²

Los resultados de este estudio tienen una significativa implicancia en la evaluación y el tratamiento de los pacientes que enfrentan una cirugía mayor no cardiaca. El perfil clínico basal de este grupo de pacientes permite discriminar inicialmente aquellos que presentan importantes factores de riesgo y tienen una alta probabilidad de presentar eventos isquémicos (15-25\%) de aquellos que no los presentan y que son de bajo riesgo ( $<3 \%$ de eventos). Estudios recientes alientan el uso de métodos de detección de isquemia no invasivos en pacientes considerados de riesgo intermedio 0 alto ${ }^{3-4}$, pero no esta demostrado que la evaluación angiográfica y la revascularización mejoren el pronostico de los pacientes con un test positivo sometidos a una cirugía mayor no cardiaca. El uso efectivo de los beta bloqueantes como lo demuestra este estudio parecería ser mas útil en la prevención de eventos perioperatorios en pacientes considerados de alto riesgo sometidos a cirugía mayor no cardiaca.

\section{Dr. Pablo F. Oberti}

Servicio de Cardiología. Hospital Italiano de Buenos Aires.

\section{Referencias}

1. Eagle KA, Brundage BH, Chaitman BR, et al. Guidelines for perioperative cardiovascular evaluation for noncardiac surgery: report of the American College of Cardiology/American Heart Association Task Force on Practice Guidelines. J Am Coll Cardiol 1996;27:910-48.

2. Mangano DT, Layug EL, Wallace A, Tateo I for the Multicenter Study of Perioperative Ischemia Research Group. Effect of atenoloL on mortality and cardiovascular morbidity after noncardiac surgery. N Engl J Med 1996;335:1713-20.

3. Sicari R, Ripoli A, Picano E, et al. on behalf of the EPIC Study Group. Perioperative prognostic value of dipyridamole echocardiography in vascular surgery. Circulation 1999; $100: I I-269-$ II-274.

4. Poldermans D, Arnese M, Fioretti PM, et al. Sustained prognostic value of dobutamine stress echocardiography for Late cardiac events after major noncardiac vascular surgery. Circulation 1997;95:53-58. 